\title{
Cosmological implications of high-energy neutrino emission from the decay of long-lived particle
}

\author{
Yohei Ema, Ryusuke Jinno and Takeo Moroi \\ Department of Physics, University of Tokyo, \\ Tokyo 113-0033, Japan \\ E-mail: ema@hep-th.phys.s.u-tokyo.ac.jp, \\ jinno@hep-th.phys.s.u-tokyo.ac.jp, moroi@hep-th.phys.s.u-tokyo.ac.jp
}

ABSTRACT: We study cosmological scenarios in which high-energy neutrinos are emitted from the decay of long-lived massive particles at the cosmic time later than a redshift of $\sim 10^{6}$. The high-energy neutrino events recently observed by the IceCube experiment suggest a new source of high-energy cosmic-ray neutrinos; decay of a heavy particle can be one of the possibilities. We calculate the spectrum of the high-energy neutrinos emitted from the decay of long-lived particles, taking account of the neutrino scattering processes with background neutrinos. Then, we derive bounds on the scenario using the observation of high-energy cosmic-ray neutrino flux. We also study constraints from the spectral distortions of the cosmic microwave background and the big-bang nucleosynthesis. In addition, we show that the $\mathrm{PeV}$ neutrinos observed by the IceCube experiment can originate from the decay of a massive particle with its mass as large as $O\left(10^{10} \mathrm{GeV}\right)$.

KeYwords: Cosmology of Theories beyond the SM, Neutrino Physics

ARXiv EPRINT: 1408.1745 


\section{Contents}

1 Introduction 1

2 Effects of neutrino emission $\quad 2$

2.1 Evolution of neutrino flux 2

2.2 CMB spectral distortions 9

$\begin{array}{lll}2.3 & \text { Effects on the BBN } & 12\end{array}$

3 Constraints on neutrino emission $\quad 12$

$\begin{array}{lll}3.1 & \text { Observational constraints } & 13\end{array}$

$\begin{array}{ll}3.2 & \text { Upper bounds on } Y_{X} \\ \end{array}$

4 Implication for recent IceCube result $\quad 16$

5 Conclusions and discussion $\quad 18$

\section{Introduction}

In large classes of particle-physics models, there exist massive long-lived particles. Even though they may not be accessed by the currently available colliders, information about those particles may be obtained from astrophysical and cosmological observations. If they are produced in the early universe and also if they decay in or near the present epoch, their decay products may affect the fluxes of high-energy cosmic rays, resulting in constraints on their relic densities, lifetimes, decay modes, and so on. In addition, models with long-lived particles have been attracted attentions to explain the results of cosmic-ray observations [1]. In particular, implications of the decay processes into $\gamma, e^{ \pm}$, and (anti-) proton have been extensively studied.

With the successful detections of high-energy cosmic-ray neutrino events at IceCube $[2$, $3]$, our understanding about the cosmic-ray neutrino flux is also significantly improving. In particular, the IceCube collaboration claims that the cosmic-ray neutrino flux in the sub$\mathrm{PeV}$ to $\mathrm{PeV}$ region is well above that of expected backgrounds, which suggests a new source of high-energy cosmic-ray neutrinos. After the IceCube results are released, it has been discussed that the decay of heavy particles may be responsible for the IceCube events [411]. ${ }^{1}$ Importantly, the lifetime of the long-lived particle (potentially) responsible for the IceCube events can be either longer or shorter than the present cosmic time. In particular, the present authors have argued that the decay of a long-lived particle (called $X$ ) in the past can be the origin of the high-energy neutrinos observed by IceCube [4]. We call this

\footnotetext{
${ }^{1}$ For other explanations for the IceCube result, including astrophysical ones, see the review [12] and the references therein.
} 
scenario "early-decay scenario," in contrast to the ones with decaying dark matter. In the previous study, we have calculated the neutrino flux originating from the decay of $X$ for the case where the neutrino scattering processes with background neutrinos are negligible (which is the case when the decay of $X$ occurs at the epoch of $1+z \lesssim 10^{4}$, with $z$ being the redshift), and have pointed out that the IceCube events may be well explained in this scenario.

In this paper, we extend our previous study and discuss astrophysical and cosmological constraints on the early-decay scenario. We pay particular attention to the effects of the neutrino scattering processes with background neutrinos, which are not completely taken into account in our previous study. We calculate the flux of cosmic-ray neutrinos originating from the decay of $X$. Then, comparing the result with the observed cosmic-ray neutrino flux, we derive an upper bound on the primordial abundance of $X$. In addition, photons and charged particles are also produced in association with the neutrino scattering processes; they result in the spectral distortion of the cosmic microwave background $(\mathrm{CMB})$ and the change of the light-element abundances produced by the big-bang nucleosynthesis (BBN), from which we obtain an upper bound on the abundance of $X$. For the study of the constraints from the CMB distortion, we take into account the present bound (COBE/FIRAS [13, 14]), or the expected bound in the future (for example, PIXIE [15] and PRISM [16]). In our study, we will not specify the detailed particle-physics model which contains the candidate of $X$, but we perform our analysis as general as possible. We discuss the constraints on the scenario using the properties of $X$, i.e., its lifetime, energy distribution of the final-state neutrinos (which is assumed to be monochromatic in this paper), and its primordial relic density. We also discuss the implication of the IceCube result in light of the early-decay scenario. In particular, it may be possible that the neutrino excess in sub-PeV region and the possible cutoff around $\mathrm{PeV}$ are simultaneously explained if we take into account the effect of the neutrinos scattered by the background neutrinos.

The organization of this paper is as follows. In section 2 we discuss the evolution of the neutrino flux originating from the massive decaying particle $X$. The effects of the produced neutrinos on the CMB distortions and the light-element abundances are also explained there. Then, in the following section, we give constraints on the primordial relic density of $X$ using the observation of the cosmic-ray neutrino flux, the CMB distortions and the light-element abundances produced by the BBN. In section 4 we discuss possible interpretations of the recent IceCube high-energy neutrino events in our scenario. The final section is devoted to the conclusions and discussion.

\section{Effects of neutrino emission}

\subsection{Evolution of neutrino flux}

Let us first discuss the evolution of the neutrino flux produced by the decay of the parent particle $X$. Once produced, the neutrinos propagate in the expanding universe scattering off background particles (in particular, neutrinos). Then, in order to obtain the neutrino flux in $l$-th flavor, $\Phi_{\nu, l}(t, E)$, which is related to the number density of the $l$-th flavor 
neutrino as $n_{\nu, l}(t)=\int d E \Phi_{\nu, l}(t, E)$, we solve the following Boltzmann equation:

$$
\begin{aligned}
\left(\frac{\partial}{\partial t}+2 H-H E \frac{\partial}{\partial E}\right) \Phi_{\nu, l}(t, E)= & -\gamma_{\nu, l}(t ; E) \Phi_{\nu, l}(t, E) \\
& +\int d E^{\prime} \Phi_{\nu, n}\left(t, E^{\prime}\right) \frac{d \gamma_{\nu, n m}\left(t ; E^{\prime}, E\right)}{d E} P_{m l}(t, E) \\
& +S_{\nu, m}(t, E) P_{m l}(t, E),
\end{aligned}
$$

where $H$ is the expansion rate of the universe, and $S_{\nu, l}(t, E)$ is the source term. In addition $\gamma_{\nu, l}(t ; E)$ is the scattering rate, and $d \gamma_{\nu, m l}\left(t ; E^{\prime}, E\right) / d E$ is the (differential) neutrino production rate with $E^{\prime}$ and $E$ being the energies of initial- and final-state neutrinos. (Here, $m$ and $n$ are flavor indices; summation over these indices is implicit.) At the cosmic time when the neutrino scattering processes become effective, at which the scattering rate becomes important, $\gamma_{\nu, l}(t ; E)$ is (almost) flavor-independent. Thus, we take $\gamma_{\nu, l}(t ; E)=\gamma_{\nu}(t ; E)$. In our calculation, the effect of the neutrino oscillation is taken into account by introducing the "transition probability" $P_{m l}(t, E)$. We approximate that the flavors are fully mixed in the case where the time scale of the neutrino oscillation (i.e., $2 E /\left|\Delta m_{21}\right|^{2}$ or $2 E /\left|\Delta m_{31}\right|^{2}$ ) is shorter than the mean free time (i.e., $\gamma_{\nu}^{-1}$ ), and that the effect of the neutrino oscillation is negligible in the opposite case. (Here, $\left|\Delta m_{21}\right|^{2}$ and $\left|\Delta m_{31}\right|^{2}$ are the neutrino mass squared differences.) Then, taking $\left|\Delta m_{21}\right|^{2}=7.50 \times 10^{-5} \mathrm{eV}^{2},\left|\Delta m_{31}\right|^{2}=2.47 \times 10^{-3} \mathrm{eV}^{2}$, $\sin ^{2} \theta_{12}=0.30, \sin ^{2} \theta_{13}=0.023$, and $\sin ^{2} \theta_{23}=0.41$ [17] with $\theta$ 's being the mixing angles in the neutrino mixing matrix, ${ }^{2} P_{m l}(t, E)$ is evaluated as follows:

1. When $\left|\Delta m_{31}\right|^{2} / 2 E<\gamma_{\nu}(t ; E)$, the scattering time scale is shorter than those of neutrino oscillation. In this case, the effect of neutrino oscillation is neglected and we take $P_{m l}(t, E)=\operatorname{diag}(1,1,1)$.

2. When $\left|\Delta m_{21}\right|^{2} / 2 E<\gamma_{\nu}(t ; E)<\left|\Delta m_{31}\right|^{2} / 2 E$, the neutrino oscillation due to $\Delta m_{21}$ is neglected, while the oscillation due to $\Delta m_{31}$ is taken into account. In this case, we take:

$$
\begin{aligned}
& P_{e \mu}(t, E)=0.02, \\
& P_{e \tau}(t, E)=0.03, \\
& P_{\mu \tau}(t, E)=0.47 .
\end{aligned}
$$

3. When $\gamma_{\nu}(t ; E)<\left|\Delta m_{21}\right|^{2} / 2 E$, we approximate that neutrino oscillations due to $\Delta m_{21}$ and $\Delta m_{31}$ are so fast that the full mixing of the neutrino flavors is realized. In this case, we take:

$$
\begin{aligned}
& P_{e \mu}(t, E)=0.28, \\
& P_{e \tau}(t, E)=0.16, \\
& P_{\mu \tau}(t, E)=0.37 .
\end{aligned}
$$

\footnotetext{
${ }^{2}$ In our approximation, $P_{m l}(t, E)$ is evaluated at the time of the neutrino emission. Therefore, if a sizable amount of neutrino propagated from the epochs of 1 or 2 to the present epoch without being scattered, we would fail to include the effects of neutrino oscillation during the propagation. However in reality, $\gamma_{\nu}(t ; E)<\left|\Delta m_{21}\right|^{2} / 2 E$ for $\tau(t, E /(1+z(t))) \lesssim 10$, where $\tau(t, E /(1+z(t)))$ is the optical depth of neutrino defined in eq. (2.13), and such a problem does not occur.
} 
Here, we neglect the $C P$-violation in the neutrino mixing, and hence we take $P_{m l}(t, E)=$ $P_{l m}(t, E)$. The diagonal elements of $P_{m l}(t, E)$ can be evaluated by using $\sum_{m} P_{l m}(t, E)=1$.

In order to take into account the effects of neutrino scattering, we consider the following scattering processes with background (anti-) neutrinos: ${ }^{3}$

- $\nu_{l}+\nu_{l, \mathrm{BG}} \rightarrow \nu_{l}+\nu_{l}$,

- $\nu_{l}+\nu_{l^{\prime}, \mathrm{BG}} \rightarrow \nu_{l}+\nu_{l^{\prime}}$, with $l \neq l^{\prime}$,

- $\nu_{l}+\bar{\nu}_{l, \mathrm{BG}} \rightarrow \nu_{l}+\bar{\nu}_{l}$,

- $\nu_{l}+\bar{\nu}_{l, \mathrm{BG}} \rightarrow l+\bar{l}$,

- $\nu_{l}+\bar{\nu}_{l, \mathrm{BG}} \rightarrow f+\bar{f}$, with $f \neq l, \nu_{l}$,

- $\nu_{l}+\bar{\nu}_{l^{\prime}, \mathrm{BG}} \rightarrow \nu_{l}+\bar{\nu}_{l^{\prime}}$, with $l \neq l^{\prime}$,

- $\nu_{l}+\bar{\nu}_{l^{\prime}, \mathrm{BG}} \rightarrow l+\bar{l}^{\prime}$, with $l \neq l^{\prime}$,

where $l=e, \mu, \tau$, while $f$ denotes the standard-model fermions, and the subscript "BG" is used for background neutrinos. The scattering rate $\gamma_{\nu}(t ; E)$ is calculated with taking into account the effects of these processes.

In the calculation of the neutrino production rate, we include two contributions as

$$
\frac{d \gamma_{\nu, m l}}{d E}=\frac{d \gamma_{\nu, m l}^{(\mathrm{dir})}}{d E}+\frac{d \gamma_{\nu, m l}^{(\gamma \gamma)}}{d E} .
$$

One is the neutrinos directly produced by the scattering processes listed above, which corresponds to the first term of the right-hand side of eq. (2.4). In the neutrino-neutrino scattering processes, energetic neutrinos are produced directly or by the decay of finalstate particles. (Notice that the standard-model fermions other than neutrinos and $e^{ \pm}$ undergo hadronization and/or decay processes after the production.) In our numerical calculation, we calculate the energy distributions of the neutrinos (as well as other stable particles, i.e, $e^{ \pm}, \gamma, p$ and $\bar{p}$ ) produced by the scattering processes listed above using PYTHIA package $[18,19]$. The other is the neutrinos produced by double-photon pair creations of standard-model fermions (the second term of the right-hand side of eq. (2.4)). A sizable amount of high-energy photons may be produced as a consequence of neutrinoneutrino scattering processes (after the hadronization and/or decay of colored particles). In addition, high-energy $e^{ \pm}$s produced by the neutrino scattering processes are converted to high-energy photons via the inverse Compton process. By scattering off the CMB, those high-energy photons may induce double-photon pair creations of standard-model fermions whose decay products contain neutrinos. Then, we estimate

$$
\frac{d \gamma_{\nu, m l}^{(\gamma \gamma)}\left(E^{\prime}, E\right)}{d E}=\int d \epsilon\left(\frac{d \gamma_{\gamma, m}\left(E^{\prime}, \epsilon\right)}{d \epsilon}+\frac{d \gamma_{e^{ \pm}, m}\left(E^{\prime}, \epsilon\right)}{d \epsilon}\right) \frac{d N_{\nu, l}^{(\gamma \gamma)}(\epsilon, E)}{d E},
$$

\footnotetext{
${ }^{3}$ If the energy of the injected neutrino is very high, the scattering process with background photons also becomes relevant. However, we have checked that the following results do not change even if we take such a process into account.
} 
where $d \gamma_{\gamma, m} / d \epsilon$ and $d \gamma_{e^{ \pm}, m} / d \epsilon$ are (differential) production rate of photon and $e^{ \pm}$via the neutrino scattering processes, respectively. (Here, $\epsilon$ denotes the energy of $\gamma$ or $e^{ \pm}$ produced by the neutrino scattering processes.) We approximate that the energy of the photon produced by the inverse Compton scattering is equal to that of the initial-state $e^{ \pm}$. This is because, in the center-of-mass frame, the inverse Compton scattering is significantly enhanced for backward scattering in the relativistic limit [20]. In addition, $d N_{\nu, l}^{(\gamma \gamma)} / d E$ is the spectrum of neutrinos (after the hadronization and/or the decay processes) produced as a consequence of the (multiple) double-photon pair creation. Using the fact that, in the center-of-mass frame, the double-photon pair creation cross section is sharply peaked when the momenta of final-state fermions are parallel (or anti-parallel) to those of initial-state photons [20], we approximate that the energy of one of the final-state fermions is equal to that of initial-state high-energy photon while that of another fermion is negligibly small. Thus, with the injections of photon and $e^{ \pm}$, electromagnetic cascade occurs. During the cascade, the energy of the electromagnetic sector is reduced via the emission of neutrino, which is due to the decay of unstable particles like muon. We approximate that the doublephoton pair productions of the fermions other than $e^{ \pm}$become ineffective for the photon with the energy $E$ once the ratio of the scattering rates $\Gamma_{\gamma \gamma \rightarrow \mu^{+} \mu^{-}} / \Gamma_{\gamma \gamma \rightarrow e^{+} e^{-}}$becomes smaller than $m_{e}^{2} / E T$ (with $m_{e}$ being the electron mass); here, we use the fact that $E_{\mathrm{i}}-E_{\mathrm{f}} \sim$ $O\left(m_{e}^{2} / T\right)$, where $E_{\mathrm{i}}$ and $E_{\mathrm{f}}$ are energies of energetic initial- and final-state particles in the processes $\gamma \gamma \rightarrow e^{+} e^{-}$and $e^{ \pm} \gamma \rightarrow e^{ \pm} \gamma$. The remaining electromagnetic particles may also affect the CMB spectrum and the light-element abundances, as we will discuss in section 2.2 and 2.3 , respectively.

When the neutrinos are produced by the decay of $X$, the source term is given by

$$
S_{\nu, l}(t, E)=\frac{1}{4 \pi} \frac{n_{X}(t)}{\tau_{X}} \frac{d N_{\nu, l}^{(X)}}{d E},
$$

where $n_{X}(t)$ is the number density of $X, \tau_{X}$ is the lifetime of $X$, and $d N_{\nu, l}^{(X)} / d E$ is the energy distribution of the $l$-th flavor neutrinos produced by the decay of $X$. Using the so-called yield variable $Y_{X}$ defined as

$$
Y_{X} \equiv\left[\frac{n_{X}(t)}{s(t)}\right]_{t \ll \tau_{X}},
$$

with $s(t)$ being the entropy density, $n_{X}(t)$ is given by

$$
n_{X}(t)=Y_{X} s(t) e^{-t / \tau_{X}}
$$

For simplicity, we consider only the case where the neutrinos produced by the decay of $X$ are monochromatic (with the energy of $\bar{E}_{\nu}$ ). Then, $d N_{\nu, l}^{(X)} / d E$ is given by

$$
\frac{d N_{\nu, l}^{(X)}}{d E}=\bar{N}_{\nu, l} \delta\left(E-\bar{E}_{\nu}\right),
$$

where $\bar{N}_{\nu, l}$ is the number of $l$-th flavor neutrinos produced by the decay of one $X$. For simplicity, we consider the case where the decay of $X$ produces equal amount of $e, \mu$, and 


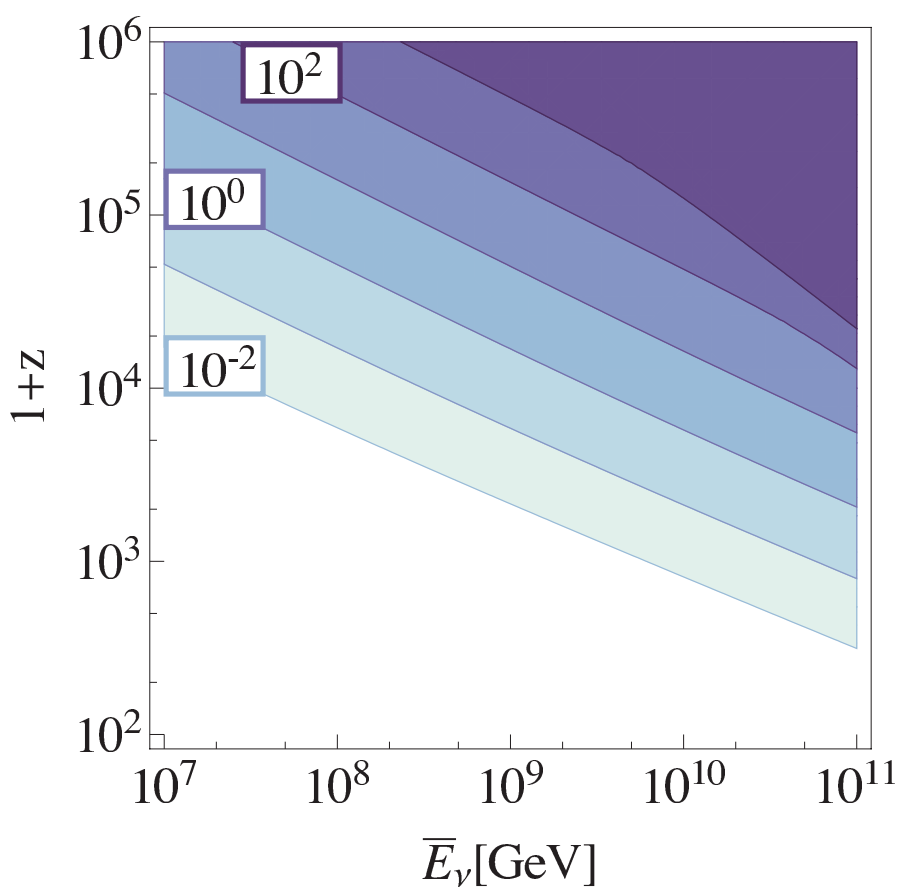

Figure 1. Contours of constant $\tau(z ; E)$, with $E$ being the present energy of the neutrino. The contours are $\tau=0.01,0.1, \cdots$, and 1000, from bottom to top. (The numbers in the figure give the value of $\tau$.) The horizontal axis is the initial energy of the neutrino $\bar{E}_{\nu}=(1+z) E$, while the vertical axis is the redshift $1+z$.

$\tau$ neutrinos, taking $\bar{N}_{\nu, e}=\bar{N}_{\nu, \mu}=\bar{N}_{\nu, \tau}=1 / 3{ }^{4}$ Moreover, we also assume that the $C P$ violation is negligible in the decay of $X$ and that the fluxes of neutrinos and anti-neutrinos are equal. We note that particles other than neutrinos such as electrons and photons may also be produced by the decay of $X$. The cosmological implications of such particles are discussed, for example, in [21-31]. Thus, in our analysis, the present neutrino flux is determined by the following three parameters:

$$
\bar{E}_{\nu}, z_{*} \equiv z\left(\tau_{X}\right), Y_{X}
$$

The neutrino flux at the present cosmic time $t_{0}$ can be decomposed into two contributions:

$$
\Phi_{\nu, l}\left(t_{0}, E\right)=\Phi_{\nu, l}^{(\text {prim) }}(E)+\Phi_{\nu, l}^{(\mathrm{sec})}(E) .
$$

Here, $\Phi_{\nu, l}^{(\text {prim) }}(E)$ is the flux of neutrinos which propagate to the present epoch without being scattered (which we call "primary neutrinos"), while $\Phi_{\nu, l}^{(\mathrm{sec})}(E)$ is that of secondary neutrinos produced by the neutrino scattering processes and the electromagnetic cascade.

\footnotetext{
${ }^{4}$ We have checked that, because of the neutrino oscillation, the resultant neutrino flux for each flavor does not depend much on this assumption.
} 
With the monochromatic neutrino injection, $\Phi_{\nu, l}^{(\text {prim })}(E)$ is given by ${ }^{5}$

$$
\Phi_{\nu, l}^{(\operatorname{prim})}(E)=\frac{1}{4 \pi} \frac{\bar{N}_{\nu, l} Y_{X} s\left(t_{0}\right)}{\tau_{X} E}\left[\frac{e^{-\bar{t} / \tau_{X}} e^{-\tau(z(\bar{t}) ; E)}}{H(\bar{t})}\right]_{1+z(\bar{t})=\bar{E}_{\nu} / E}
$$

where $\tau(z ; E)$ is the optical depth of neutrinos

$$
\tau(z ; E) \equiv \int_{t(z)}^{t_{0}} d t^{\prime} \gamma_{\nu}\left(t^{\prime} ;\left(1+z\left(t^{\prime}\right)\right) E\right)
$$

From eq. (2.12), one can see that the neutrinos produced at higher redshifts contribute to the present flux at lower energies.

In order to see when the neutrino scattering is effective, in figure 1, we plot the contours of constant $\tau(z ; E)$ on $\bar{E}_{\nu} \equiv(1+z) E$ vs. $1+z$ plane. As one can see, the optical depth increases as $\bar{E}_{\nu}$ or $z$ becomes larger; this is because the neutrino scattering cross section is more enhanced with higher center-of-mass energy $E_{\mathrm{CM}}$ (as far as $E_{\mathrm{CM}}<m_{Z}$ ). The optical depth becomes $\sim 1$ when the scattering rate of the neutrino is comparable to the expansion rate of the universe. For the present energy of $E=10^{6}$ and $10^{7} \mathrm{GeV}$, for example, $\tau(z ; E) \gtrsim 1$ is realized when $1+z \gtrsim 10^{4}$ and $3 \times 10^{3}$, respectively. When $\tau \gtrsim 1$, the neutrino scattering processes become important and $\Phi_{\nu, l}^{(\mathrm{sec})}(E)$ is sizable. One can also see that some of the contours show bending behavior. This is due to the change in the neutrino cross section at $E_{\mathrm{CM}} \sim m_{Z}$.

We numerically evaluate the neutrino flux by solving eq. (2.1). For this purpose, we introduce the Green's function $G_{m l}\left(t^{\prime}, E^{\prime} ; t, E\right)$, which satisfies

$$
\begin{aligned}
&\left(\frac{\partial}{\partial t}+2 H\right.\left.-H E \frac{\partial}{\partial E}\right) G_{m l}\left(t^{\prime}, E^{\prime} ; t, E\right) \\
&=-\gamma_{\nu}(t ; E) G_{m l}\left(t^{\prime}, E^{\prime} ; t, E\right)+\int d E^{\prime \prime} G_{m n}\left(t^{\prime}, E^{\prime} ; t, E^{\prime \prime}\right) \frac{d \gamma_{\nu, n p}\left(t ; E^{\prime \prime}, E\right)}{d E} P_{p l}(t, E) \\
& \quad+\delta_{m l} \delta\left(t^{\prime}-t\right) \delta\left(E^{\prime}-E\right)
\end{aligned}
$$

and

$$
G_{m l}\left(t^{\prime}, E^{\prime} ; t, E\right)_{t^{\prime}>t}=0
$$

With the Green's function, the neutrino flux is given by

$$
\Phi_{\nu, l}(t, E)=\int_{0}^{t} d t^{\prime} \int d E^{\prime} S_{m}\left(t^{\prime}, E^{\prime}\right) P_{m n}\left(t^{\prime}, E^{\prime}\right) G_{n l}\left(t^{\prime}, E^{\prime} ; t, E\right) .
$$

\footnotetext{
${ }^{5}$ The effects of neutrino oscillation do not appear in eq. (2.12) because we assume that the neutrinos produced by the decay of $X$ are flavor-universal.
} 
In order to evaluate $G_{m l}\left(t^{\prime}, E^{\prime} ; t, E\right)$, we use the fact that $G_{m l}\left(t^{\prime}, E^{\prime} ; t, E\right)$ satisfies the following relation:

$$
\begin{aligned}
G_{m l}\left(t^{\prime}, E^{\prime} ; t, E\right)=\theta\left(t-t^{\prime}\right)\left[\left(\frac{a\left(t^{\prime}\right)}{a(t)}\right)^{2} e^{-\tilde{\tau}\left(t^{\prime}, t ; E^{\prime}\right)} \delta_{m l} \delta\left(E^{\prime}-a(t) E / a\left(t^{\prime}\right)\right)\right. & \\
& +\int_{t^{\prime}}^{\infty} d t^{\prime \prime} \int d E^{\prime \prime}\left(\frac{a\left(t^{\prime}\right)}{a\left(t^{\prime \prime}\right)}\right)^{3} e^{-\tilde{\tau}\left(t^{\prime}, t^{\prime \prime} ; E^{\prime}\right)} \times \\
& \left.\times \frac{d \gamma_{\nu, m n}\left(t^{\prime \prime} ; a\left(t^{\prime}\right) E^{\prime} / a\left(t^{\prime \prime}\right), E^{\prime \prime}\right)}{d E^{\prime \prime}} P_{n p}\left(t^{\prime \prime}, E^{\prime \prime}\right) G_{p l}\left(t^{\prime \prime}, E^{\prime \prime} ; t, E\right)\right],
\end{aligned}
$$

where $a(t)$ is the scale factor at the cosmic time $t$, and

$$
\tilde{\tau}\left(t^{\prime}, t ; E^{\prime}\right) \equiv \int_{t^{\prime}}^{t} d t^{\prime \prime} \gamma_{\nu}\left(t^{\prime \prime} ; a\left(t^{\prime}\right) E^{\prime} / a\left(t^{\prime \prime}\right)\right)
$$

In our numerical calculation, we discretize eq. (2.17) and recursively evaluate the Green's function, with which the neutrino flux is calculated. When the neutrinos produced by the decay of $X$ are flavor-universal and neutrino oscillation is taken into account, the present neutrino fluxes are almost flavor-universal. Therefore, in the following discussion, we neglect the flavor dependence of the neutrino flux and use $\Phi_{\nu}(E)$, which is defined as

$$
\Phi_{\nu}(E) \equiv \frac{1}{3}\left[\Phi_{\nu, e}\left(t_{0}, E\right)+\Phi_{\nu, \mu}\left(t_{0}, E\right)+\Phi_{\nu, \tau}\left(t_{0}, E\right)\right] .
$$

In figure 2, we show the neutrino fluxes at the present epoch for several values of $z_{*}$. Here, we take $Y_{X}=10^{-26}$ and $\bar{E}_{\nu} /\left(1+z_{*}\right)=1 \mathrm{PeV}$. The qualitative behavior of the neutrino spectrum can be understood as follows:

1. With small enough $z_{*}$, the neutrino scattering is inefficient. In such a case, the neutrino spectrum is affected only by the redshift and has a peak at $E \sim \bar{E}_{\nu} /\left(1+z_{*}\right)$. (See the top-left panel with $1+z_{*}=10^{2}$.)

2. With the increase of $z_{*}$, a tail-like structure shows up because of the neutrino scattering processes. (See the top-right panel with $1+z_{*}=2 \times 10^{3}$.) Comparing with the top-left panel, we can also see that the flux is slightly reduced at $2 \times 10^{5} \mathrm{GeV} \lesssim E \lesssim 5 \times 10^{5} \mathrm{GeV}$ in the top-right panel. This is due to the fact that the neutrinos with lower present energies are more likely to be affected by the scattering processes with the background neutrinos because they are produced at higher redshifts. In the top-right panel, the neutrino scattering thus reduces the flux at $2 \times 10^{5} \mathrm{GeV} \lesssim E \lesssim 5 \times 10^{5} \mathrm{GeV}$, while the secondary neutrinos produced by the neutrino scattering and the electromagnetic cascade contribute to the flux at $E \lesssim 2 \times 10^{5} \mathrm{GeV}$.

3. With larger $z_{*}, \bar{E}_{\nu}$ and $z_{*}$ become so large that a sizable fraction of neutrinos experience the scatterings with background neutrinos. Consequently, the neutrino flux at around the peak is also reduced. (See the bottom-left panel with $1+z_{*}=10^{4}$.) 

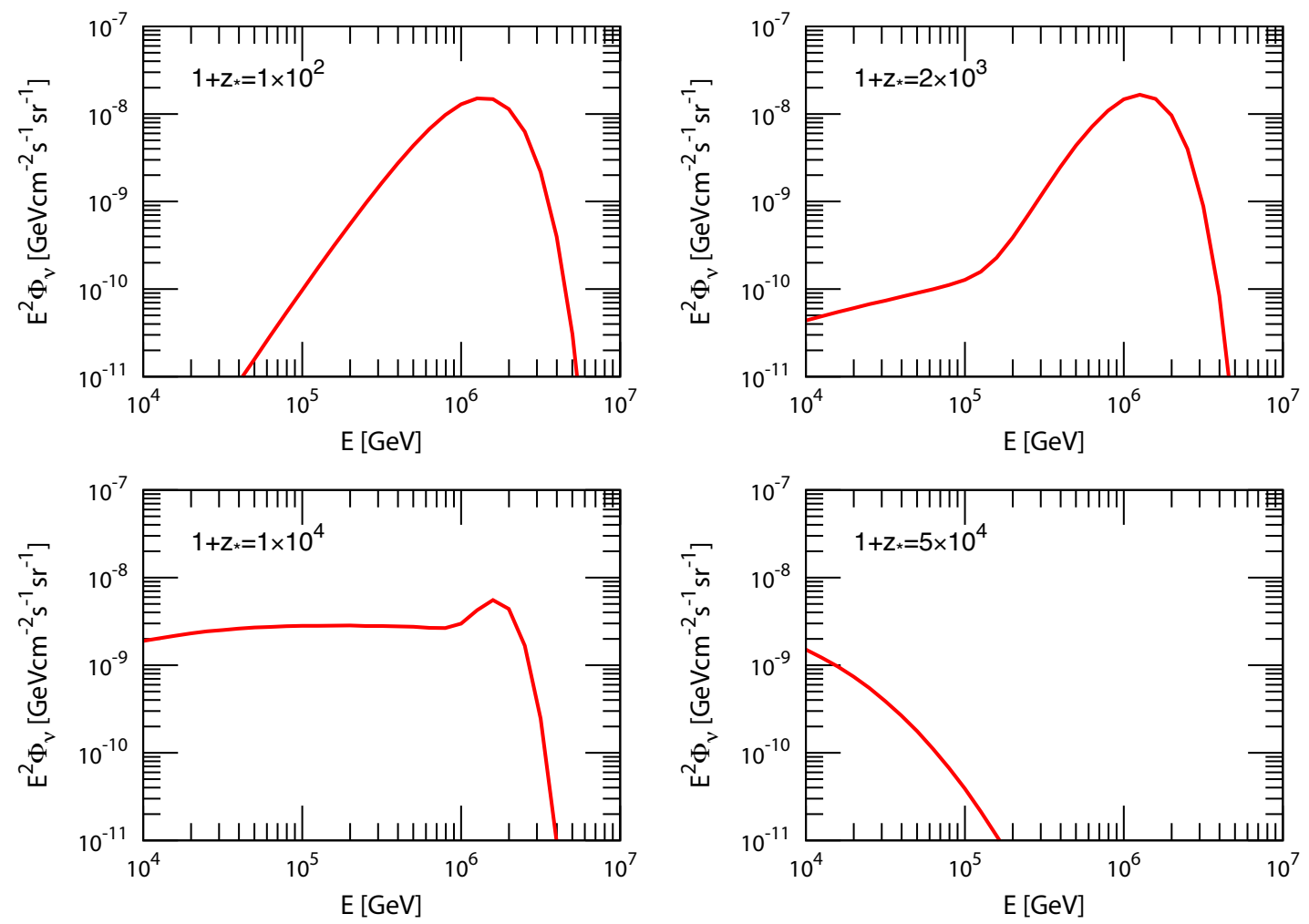

Figure 2. The present per-flavor neutrino fluxes for some different input parameters. Top left: $\left(\bar{E}_{\nu}, 1+z_{*}, Y_{X}\right)=\left(10^{8} \mathrm{GeV}, 10^{2}, 10^{-26}\right)$. Top right: $\left(\bar{E}_{\nu}, 1+z_{*}, Y_{X}\right)=\left(2 \times 10^{9} \mathrm{GeV}, 2 \times 10^{3}, 10^{-26}\right)$. Bottom left: $\left(\bar{E}_{\nu}, 1+z_{*}, Y_{X}\right)=\left(10^{10} \mathrm{GeV}, 10^{4}, 10^{-26}\right)$. Bottom right: $\left(\bar{E}_{\nu}, 1+z_{*}, Y_{X}\right)=(5 \times$ $\left.10^{10} \mathrm{GeV}, 5 \times 10^{4}, 10^{-26}\right)$.

4. Then, with larger enough $z_{*}$, the neutrino scattering processes are so efficient that almost all the neutrinos emitted from the $X$ decay are scattered. (See the bottomright panel with $1+z_{*}=5 \times 10^{4}$.) The present neutrino flux originates from secondary neutrinos.

\subsection{CMB spectral distortions}

Next, we discuss the effects of electromagnetic particles produced by the neutrino scattering processes. In general, if photons or charged particles are injected in the early universe, these particles may affect the spectrum of the CMB. The type of the spectral distortion relevant for the present scenario depends on the epoch at which the energy injection occurs [32-37]: ${ }^{6}$

- For $z \gtrsim 2 \times 10^{6}$, the complete thermalization is achieved and there is no spectral distortion.

- For $5 \times 10^{4} \lesssim z \lesssim 2 \times 10^{6}$, the kinetic equilibrium is realized while the chemical equilibrium is not. As a result, the so-called $\mu$-type distortion is produced.

\footnotetext{
${ }^{6}$ In our analysis, we approximate that the distorted spectrum can be parametrized by $y$ and $\mu$. However, for $1.5 \times 10^{4} \lesssim z \lesssim 2 \times 10^{5}$, we might better consider intermediate-type distortions; for such an analysis of the CMB distortion, see [26, 28-31]. In addition, with such a precise analysis, we may have a chance to acquire information about the lifetime of $X$ [26, 29-31].
} 
- For $z_{\text {rec }} \lesssim z \lesssim 5 \times 10^{4}$, where $z_{\text {rec }}$ is the redshift at the recombination, even the kinetic equilibrium is not achieved. Then, the so-called $y$-type distortion occurs.

In the case of the $\mu$-type distortion, the distribution function of the CMB photon $f_{\gamma}(\omega)$ (with $\omega$ being the energy of $\gamma$ ) becomes the Bose-Einstein distribution with the chemical potential $\mu[36]$ :

$$
f_{\gamma}(\omega)=\left[\exp \left(\frac{\omega}{T}+\mu\right)-1\right]^{-1}
$$

Here, $T$ is the CMB temperature after the completion of the decay of $X$ [38]. The chemical potential is given by

$$
\mu \simeq 1.4 \int_{z_{K}}^{\infty} d z \frac{Q(z)}{\rho_{\mathrm{rad}}(z)} J_{\mu}(z)
$$

where $1+z_{K}=5 \times 10^{4}, \rho_{\text {rad }}(z)$ is the radiation energy density at the redshift $1+z$, and $Q(z)$ is the energy injection rate. In addition, $J_{\mu}$ is the so-called distortion visibility function:

$$
J_{\mu}(z)=\exp \left[-\left(\frac{z}{2 \times 10^{6}}\right)^{2.5}\right],
$$

which parametrize the fraction of injected energy at the redshift $1+z$ converted into the $\mu$-type distortion.

In the case of the $y$-type distortion, the deviation of the CMB spectrum from the black-body distribution is parametrized as [32]

$$
\frac{\delta f_{\gamma}(\omega)}{f_{\gamma}(\omega)}=y \frac{x e^{x}}{e^{x}-1}\left[x\left(\frac{e^{x}+1}{e^{x}-1}\right)-4\right]
$$

where $x \equiv \omega / T$, and the $y$ parameter is estimated as $^{7}$

$$
y \simeq \frac{1}{4} \int_{0}^{z_{K}} d z \frac{Q(z)}{\rho_{\text {rad }}(z)} .
$$

In the present scenario, $Q(z)$ comes from secondary photons and charged particles produced by the neutrino scattering processes. As we have mentioned, we calculate the energy spectra of the stable electromagnetic particles (i.e., $\gamma, e^{ \pm}, p$ and $\bar{p}$ ) using PYTHIA in order to evaluate $Q(z)$. We assume that the secondary photons and charged particles are instantaneously converted into the $y$ or $\mu$ parameters after the double-photon pair productions of the fermions other than $e^{ \pm}$become ineffective. This is a good approximation in the case of our interest because the interactions of photons and charged particles are fast enough when the neutrino scattering is effective [39].

We calculate the $y$ and $\mu$ parameters as functions of $\bar{E}_{\nu}, z_{*}$, and $Y_{X}$. In figures 3 and 4 , we show the contours of constant $y$ and $\mu$ for $Y_{X}=10^{-22}$ on $\bar{E}_{\nu}$ vs. $1+z_{*}$ plane.

\footnotetext{
${ }^{7}$ We set the lower bound of the integral in eq. (2.24) to 0 , since changing it below $z_{\text {rec }}$ does not affect our result.
} 


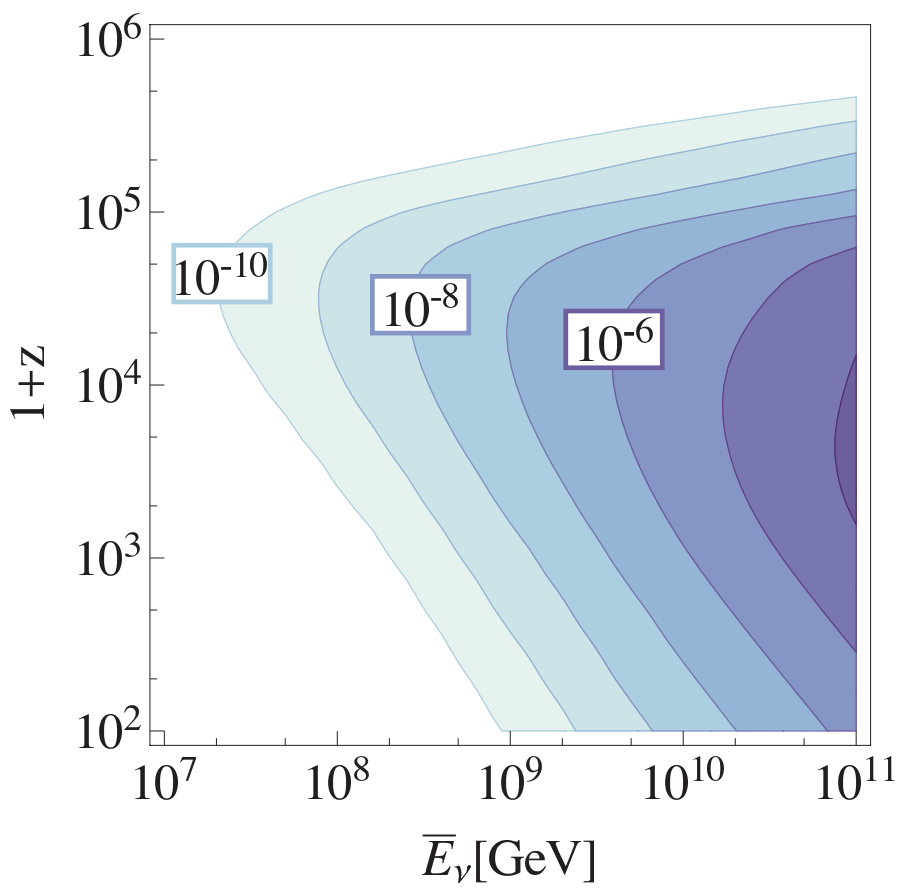

Figure 3. Contours of constant $y$ on $\bar{E}_{\nu}$ vs. $1+z_{*}$ plane. The contours are $y=10^{-10}, 10^{-9}$, $\cdots$, and $10^{-4}$, from left to right. (The numbers in the figure give the value of $y$.) Here, we take $Y_{X}=10^{-22}$.



Figure 4. Contours of constant $\mu$ on $\bar{E}_{\nu}$ vs. $1+z_{*}$ plane. The contours are $\mu=10^{-10}, 10^{-9}$, $\cdots$, and $10^{-4}$, from left to right. (The numbers in the figure give the value of $\mu$.) Here, we take $Y_{X}=10^{-22}$. 
For $E \lesssim 10^{7} \mathrm{GeV}$, both the $y$-type and $\mu$-type distortions are almost negligible. This is because neutrinos are very transparent for $E \lesssim 10^{7} \mathrm{GeV}$ and $1+z_{*} \lesssim 10^{6}$. The $y$-type and $\mu$-type distortions become important only when a significant amount of secondary photons and charged particles are produced by the neutrino scattering.

As the energy of the neutrino becomes larger, $y$ and/or $\mu$ may become sizable. For $1+z_{*} \lesssim 10^{4}, y$ is larger for larger $1+z_{*}$ or $\bar{E}_{\nu}$. This is because the neutrino scattering is more efficient with larger $1+z_{*}$ or $\bar{E}_{\nu}$. For $1+z_{*} \gtrsim 5 \times 10^{4}$, y rapidly decreases as $1+z_{*}$ increases. This is mainly due to the fact that, for $1+z_{*} \gtrsim 5 \times 10^{4}$, a large fraction of $X$ decays before $z=z_{K}$. On the contrary, the $\mu$-type distortion is important only when $1+z_{*} \gtrsim 5 \times 10^{4}$. The reason is that a significant amount of $X$ must decay when $z>z_{K}$ to realize sizable $\mu$. One can also see that, at $1+z_{*} \gtrsim 10^{5}, \mu$ becomes suppressed with the increase of $z_{*}$. For $E \gtrsim 10^{7} \mathrm{GeV}$ and $1+z_{*} \gtrsim 10^{5}, \mu$ can be approximately estimated as the ratio of the energy density of $X$ to that of radiation at $z=z_{*}$. With $\bar{E}_{\nu}$ and $Y_{X}$ being fixed, the energy density of $X$ is proportional to $(1+z)^{3}$, while that of radiation energy density scales as $(1+z)^{4}$. Therefore the ratio at $z=z_{*}$ is proportional to $\left(1+z_{*}\right)^{-1}$, resulting in the fact that $\mu$ is also proportional to $\left(1+z_{*}\right)^{-1}$ as far as $1+z_{*} \lesssim 10^{6}$.

\subsection{Effects on the BBN}

Finally, we consider the effects of the high-energy neutrino injection on the BBN. Due to the injection of hadrons and electromagnetic particles as a consequence of the scattering processes of high-energy neutrinos, hadronic and electromagnetic showers are induced. Energetic particles in the shower scatter off the light elements generated by the BBN reactions, which results in the change of light-element abundances. Using the fact that the standard BBN scenario predicts light-element abundances which are more-or-less consistent with observations, scenarios with too much injections of hadrons and electromagnetic particles are excluded.

In the following, we consider the case where $z_{*}$ is smaller than $\sim 10^{6}$, for which photodissociation processes become important. In particular, the overproduction of ${ }^{3} \mathrm{He}$ due to the dissociation of ${ }^{4} \mathrm{He}$ provides the most stringent constraint; in our analysis, we adopt the following bound $[40,41]:^{8}$

$$
E_{\mathrm{vis}} Y_{X}<2 \times 10^{-14} \mathrm{GeV},
$$

where $E_{\text {vis }}$ is the total energy injection in the form of electromagnetic particles due to the decay of one $X$. We have estimated $E_{\text {vis }}$ by using the energy injection rate at the cosmic time being $\tau_{X}$.

\section{Constraints on neutrino emission}

Now, we are at the position to derive constraints on the early-decay scenario with neutrino emission. Here, we derive upper bounds on the yield variable $Y_{X}$ by using the constraints from observations. In the present scenario, we take account of the following constraints: (i)

\footnotetext{
${ }^{8}$ For the BBN constraints on the neutrino injection with smaller $\bar{E}_{\nu}$ than the present case, see [42, 43].
} 
observational bounds on the high-energy neutrino flux, (ii) bounds from the CMB spectral distortions as we discussed in section 2.2, and (iii) bounds from the BBN as we discussed in section 2.3 .

\subsection{Observational constraints}

We first consider the constraints from the neutrino flux. To put bounds on $Y_{X}$, we adopt the following upper bounds on the neutrino flux:

(a) For $E \leq 10^{5} \mathrm{GeV}$, we take as the upper bound twice the atmospheric neutrino flux given in [44] and [45] (model 9 of figure 10 in [45]).

(b) For $10^{5} \mathrm{GeV}<E \leq 10^{6} \mathrm{GeV}$, we take $E^{2} \Phi_{\nu}(E)=3.0 \times 10^{-8}(E / 100 \mathrm{TeV})^{-0.3}$ $\mathrm{GeVcm}^{-2} \mathrm{~s}^{-1} \mathrm{sr}^{-1}$ as the upper bound. This is twice the best-fit value of the neutrino flux for this energy region given by the IceCube collaboration [3].

(c) For $10^{6} \mathrm{GeV}<E \leq 10^{10} \mathrm{GeV}$, we use the upper bound on the flux in this energy region given by the IceCube collaboration [46].

As we discussed in section 2.2, we may also obtain the bound on $Y_{X}$ from the CMB spectrum distortion. Currently, the COBE/FIRAS experiment $[13,14]$ gives the most stringent upper bounds on $y$ and $\mu$, which are

$$
|y| \leq 1.5 \times 10^{-5}
$$

and

$$
|\mu| \leq 9 \times 10^{-5}
$$

We use these values to derive constraints on the yield variable $Y_{X}$.

In addition, we consider the bound from the BBN. The discussion below takes account of the constraint given in eq. (2.25).

\subsection{Upper bounds on $Y_{X}$}

Taking accounts of the observational bounds discussed in the previous subsection, we derive the upper bound on the yield variable $Y_{X}$ as a function of $\bar{E}_{\nu}$ and $z_{*}$. In figure 5 , we plot the upper bound on $Y_{X}$. The bound comes from the neutrino flux (BBN) below (above) the yellow line; we find that the bound from the CMB distortion is currently weaker than that from BBN.

One can see that, for $1+z_{*} \lesssim 10^{4}$, the upper bound on $Y_{X}$ depends only on the combination of $\bar{E}_{\nu} /\left(1+z_{*}\right)$. This is because the neutrino scattering processes are unimportant for neutrinos produced at $1+z \lesssim 10^{4}$. Then, the neutrino flux is dominated by $\Phi_{\nu}^{(\text {prim) }}$ given in eq. (2.12), which is sensitive to the combination of $\bar{E}_{\nu} /\left(1+z_{*}\right)$. In such a region, we also note that the observational constraints on the neutrino flux (a), (b), and (c) give the most stringent bound on $Y_{X}$ for $\bar{E}_{\nu} /\left(1+z_{*}\right) \lesssim 10^{5} \mathrm{GeV}, 10^{5} \mathrm{GeV} \lesssim \bar{E}_{\nu} /\left(1+z_{*}\right) \lesssim 10^{6} \mathrm{GeV}$, and $\bar{E}_{\nu} /\left(1+z_{*}\right) \gtrsim 10^{6} \mathrm{GeV}$, respectively. This can be understood from the fact that the 


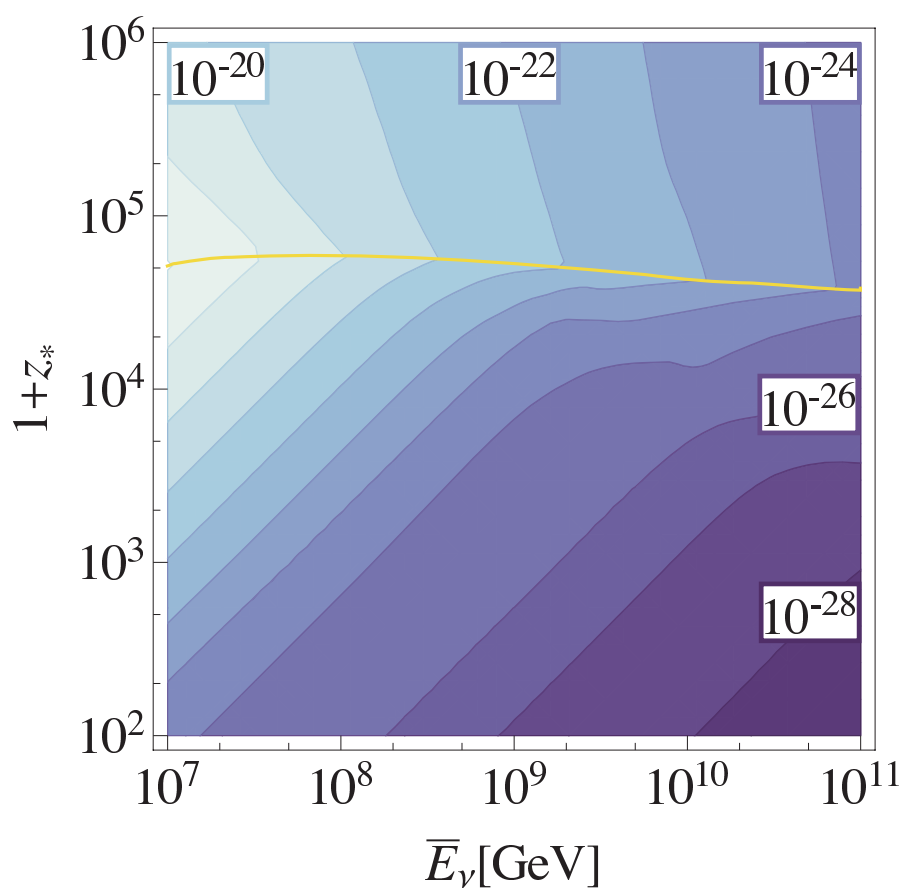

Figure 5. The upper bound on $Y_{X}$ allowed by the current observations as a function of the neutrino energy $\bar{E}_{\nu}$ at the emission and the typical redshift $z_{*}$ at the decay. The contours are the upper bound on $Y_{X}$ equal to $10^{-16}, 10^{-17}, \cdots$, and $10^{-28}$, from left to right. (The numbers in the figure give the value of the upper bound.) Below (above) the yellow line, the constraint from the high-energy neutrino flux (BBN) is stronger.

present neutrino flux has a peak at $E \sim \bar{E}_{\nu} /\left(1+z_{*}\right)$ if the effects of the neutrino scattering are negligible, as one can see from figure 2 .

One can also see that, for $\bar{E}_{\nu} \gtrsim 10^{9} \mathrm{GeV}$, the constraint on $Y_{X}$ becomes weaker at around $1+z_{*} \sim 10^{4}$. This is because the neutrino scattering processes are effective in this region, resulting in the suppression of the high-energy neutrino flux. In addition, the constraints from the $\mathrm{CMB}$ spectral distortions and the BBN are not so stringent in this region.

We also consider the prospects of testing the present scenario, paying particular attention to the possible improvement in the determination of the $y$ and $\mu$ parameters in the future. For example, the PIXIE experiment [15] will offer much better sensitivity to the CMB spectral distortions; $5 \sigma$ detection is expected when

$$
\left|y^{(\mathrm{PIXIE})}\right|=1 \times 10^{-8},
$$

or

$$
\left|\mu^{(\mathrm{PIXIE})}\right|=5 \times 10^{-8} .
$$

In figure 6 , we show the lower limit on $Y_{X}$ for the $5 \sigma$ detection with the expected PIXIE sensitivity. The shape of the contours reflects the dependences of $y$ and $\mu$ on $\left(\bar{E}_{\nu}, z_{*}\right)$ shown in figures 3 and 4 . There are kink-like structures at $1+z_{*} \sim 5 \times 10^{4}$. This is due to the fact 


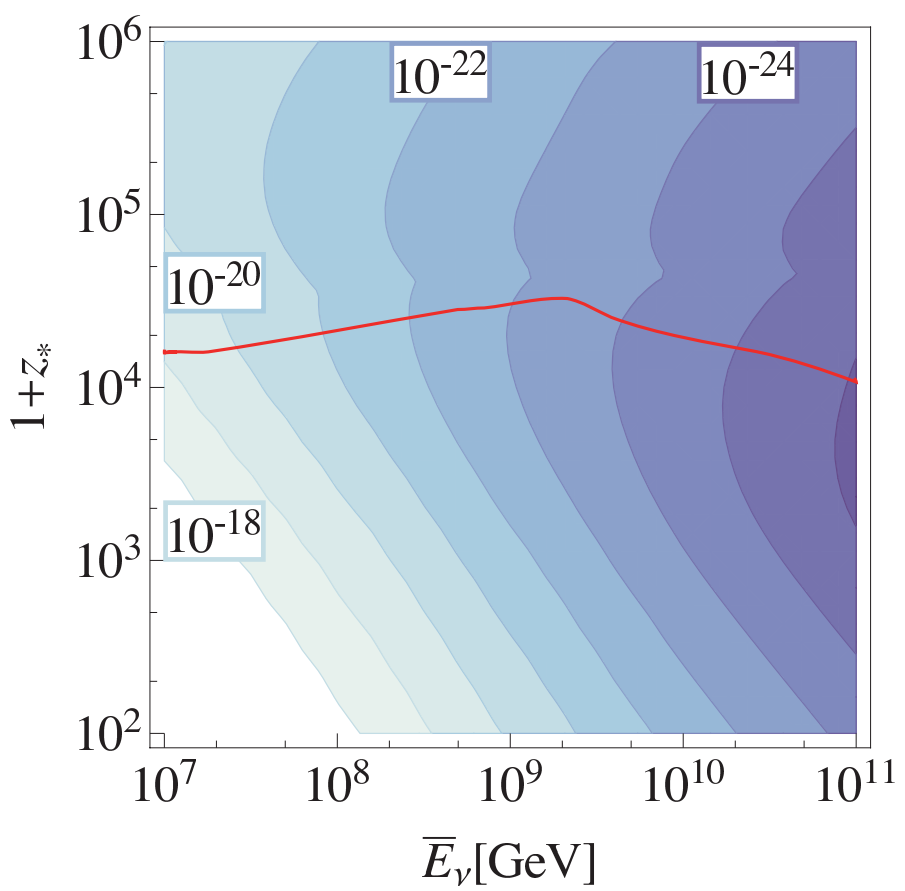

Figure 6. The lower limit on $Y_{X}$ which can be detected by the PIXIE experiment at $5 \sigma$ significance. The contours are the lower bound on $Y_{X}$ equal to $10^{-16}, 10^{-17}, \cdots$, and $10^{-28}$, from left to right. (The numbers in the figure give the value of the lower bound for the detection.) Below the red line, the values shown in the figure are already excluded by the current observations of the cosmic-ray neutrino flux.

that the injected energy is equally distributed into $y$ and $\mu$ in our approximation for such a redshift; the lowest value of $Y_{X}$ for the detection becomes slightly weaker. Then, above and below the kink, the value of $Y_{X}$ shown in figure 6 is obtained from the consideration of $\mu$ - and $y$-type distortions, respectively.

Even with the expected sensitivity of the PIXIE experiment, the current bound on the neutrino flux provides better sensitivity to the present scenario if $1+z_{*} \lesssim 2 \times 10^{4}$. In figure 6 , the boundary of such a region is indicated by the red line; in the region below the red line, the values of $Y_{X}$ shown in the figure are already excluded by the current bounds on the neutrino flux. In other words, for $1+z_{*} \approx 2 \times 10^{4}$ where the neutrino scattering is efficient, future observations of the CMB spectral distortions can test the parameter space which is not explored by the current data.

We also note here that the PRISM experiment may provide another accurate probe of the $y$ - and $\mu$-parameters. In [16], it is claimed that the sensitivity of the PRISM experiment can be as good as $\Delta \rho_{\text {rad }} / \rho_{\text {rad }} \sim O\left(10^{-9}\right)$, where $\Delta \rho_{\text {rad }}$ is the total amount of the energy release from decaying particles, which may correspond to a better sensitivity than the PIXIE experiment. With detection sensitivities other than eqs. (3.3) and (3.4), the value of $Y_{X}$ required for the detection of the signal can be obtained by rescaling the values shown in figure 6.

So far, we have assumed that $X$ dominantly decays into neutrinos. If electromagnetic particles are efficiently emitted by the decay of $X$, however, we should also consider con- 

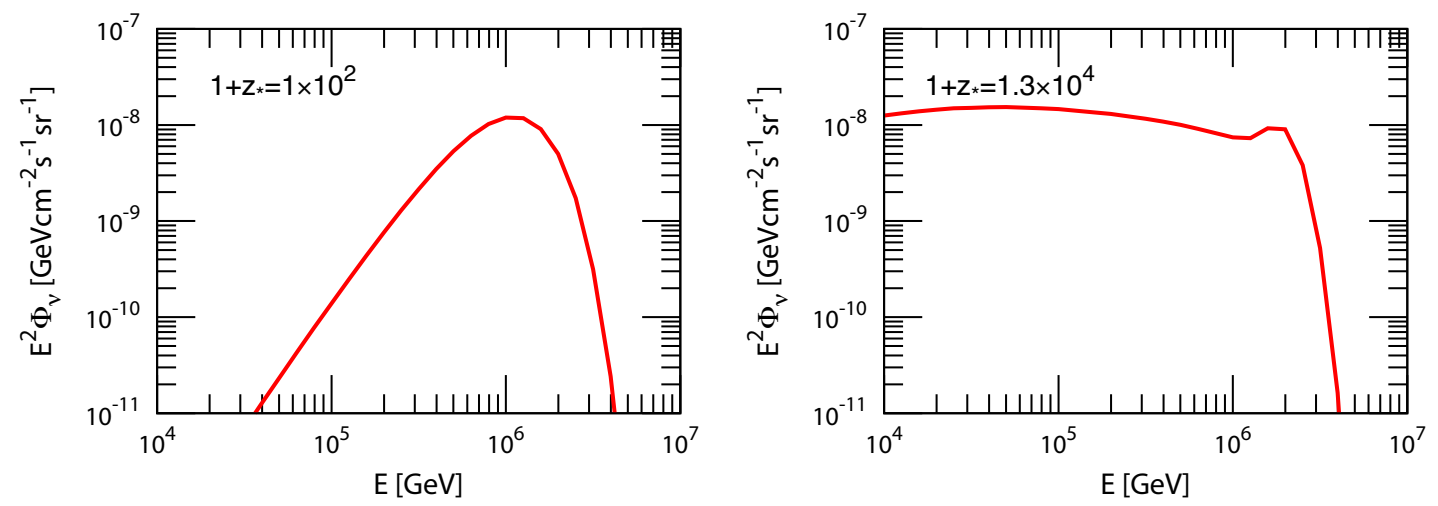

Figure 7. The present per-flavor neutrino fluxes for two different parameters. Left: $\left(\bar{E}_{\nu}, 1+\right.$ $\left.z_{*}, Y_{X}\right)=\left(8 \times 10^{7} \mathrm{GeV}, 10^{2}, 10^{-26}\right)$ Right: $\left(\bar{E}_{\nu}, 1+z_{*}, Y_{X}\right)=\left(1.3 \times 10^{10} \mathrm{GeV}, 1.3 \times 10^{4}, 5 \times 10^{-26}\right)$.

straints from these decay products. We briefly comment on such a case although it is beyond the scope of our study. When $1+z_{*} \gtrsim 2 \times 10^{3}$, the electromagnetic particles produced by $X$ contribute to the $\mu$-type and $y$-type distortions or the dissociation processes of the light elements after causing the electromagnetic cascade discussed in section 2.1. For $\tau(z ; E) \gtrsim 1$, the neutrino scattering processes are so efficient that the neutrinos produced by $X$ also induce the electromagnetic cascade. As a result, if the same amount of neutrinos and electromagnetic particles are produced by $X$, the constraint on $Y_{X}$ due to the distortion of the CMB spectrum or the light-element abundances is expected to be more-or-less unchanged for such a parameter region. For $\tau(z ; E) \lesssim 1$, however, the constraint from electromagnetic particles due to the distortion of the CMB spectrum or the light-element abundances is stronger than that from neutrinos. When $1+z_{*} \lesssim 2 \times 10^{3}$, on the contrary, electromagnetic particles produced by $X$ may change the ionization history, which also gives the constraint on the injection of electromagnetic particles [22, 25, 27].

\section{Implication for recent IceCube result}

In this section, we discuss the implications of the early-decay scenario for the explanation of the origin of the high-energy cosmic-ray neutrinos observed by the IceCube collaboration.

Recently, the IceCube collaboration has published the results of their three-year observation of high-energy neutrinos [3]. They detected three high-energy neutrino events (nicknamed as Bert, Ernie, and Big Bird) with the deposited energy of $1 \mathrm{PeV} \lesssim E \lesssim 2 \mathrm{PeV}$. The number is well above the expected background. In addition to the $\mathrm{PeV}$ neutrino events, the IceCube collaboration detected 34 events in the energy region of $30 \mathrm{TeV} \lesssim E \lesssim 1 \mathrm{PeV}$, thus finding 37 events in total. Considering that the expected background is $8.4 \pm 4.2$ from cosmic-ray muons and $6.6_{-1.6}^{+5.9}$ from atmospheric neutrinos in this energy region [3], this gap suggests a new source of the energetic cosmic-ray neutrinos. The IceCube collaboration claims that the per-flavor flux of $E^{2} \Phi_{\nu}(E)=(0.95 \pm 0.3) \times 10^{-8} \mathrm{GeVcm}^{-2} \mathrm{~s}^{-1} \mathrm{sr}^{-1}$ in the energy region of $60 \mathrm{TeV}<E<3 \mathrm{PeV}$ is consistent with the detected 37 events. ${ }^{9}$ It is also

\footnotetext{
${ }^{9}$ For the detailed analysis of the standard-model interaction of neutrino with the detector, see also [47].
} 
claimed that, if the unbroken $E^{-2}$ power law spectrum is adopted, additional 3.1 events is expected above $2 \mathrm{PeV}$, while no event is observed in this energy region. One possibility is that the neutrino spectrum obeys $\sim E^{-2}$ power law with the cutoff at the energy slightly above $\sim \mathrm{PeV}[3]$.

Because the origin of the high-energy cosmic-ray neutrino flux is yet unknown, we pursue the possibility that the decay of an exotic particle is responsible for it. We will see that the three $\mathrm{PeV}$ neutrino events at IceCube can be well explained in the present scenario. In addition, we will also see that $E^{-2}$ power law with the cutoff at a few $\mathrm{PeV}$ may be realized, since the neutrino flux at the energy higher than the position of the peak is exponentially suppressed.

In figure 7, we show the present neutrino flux for two sample points, which are given by

- Sample point 1 (left panel): $\left(\bar{E}_{\nu}, 1+z_{*}, Y_{X}\right)=\left(8 \times 10^{7} \mathrm{GeV}, 10^{2}, 10^{-26}\right)$,

- Sample point 2 (right panel): $\left(\bar{E}_{\nu}, 1+z_{*}, Y_{X}\right)=\left(1.3 \times 10^{10} \mathrm{GeV}, 1.3 \times 10^{4}, 5 \times 10^{-26}\right)$.

The neutrinos produced by $X$ are very transparent for the case of the sample point 1 . On the contrary, for the sample point 2, a sizable amount of the initial neutrinos produced by $X$ is scattered and the secondary neutrinos also contribute to the present neutrino flux. In both sample points, the flux is $E^{2} \Phi_{\nu}(E) \sim 10^{-8} \mathrm{GeVcm}^{-2} \mathrm{~s}^{-1} \mathrm{sr}^{-1}$ at around $\mathrm{PeV}$, so they may explain the IceCube $\mathrm{PeV}$ events.

In the right panel in figure 7, one can also see that the energy dependence of the flux is close to $E^{-2}$ for $E \lesssim 2 \mathrm{PeV}$. Therefore, with the parameters of our choice, there is a possibility to explain all the IceCube events in the energy region of $30 \mathrm{TeV} \lesssim E \lesssim 2 \mathrm{PeV}$ in the present scenario. It should be, however, noted that the optical depth $\tau$ is very sensitive to $\bar{E}_{\nu}$ and $z_{*}$ in the parameter region near the sample point 2. Therefore, the shape of the present neutrino flux strongly depends on these parameters. Requiring that the flux obeys a power law of $E^{-2}-E^{-2.3}$ in the energy region of $60 \mathrm{TeV}<E<3 \mathrm{PeV}$, for example, $z_{*}$ should be tuned with the accuracy of $O(10 \%)$ assuming that $\bar{E}_{\nu} \sim 10^{10} \mathrm{GeV}$. We emphasize here that, in order to realize the $\sim E^{-2}$ power law, the neutrino scattering processes should become efficient, which predicts sizable $y$ (or $\mu$ ). In the sample point 2, for example, $y=3.0 \times 10^{-9}$ (and $\mu=6.4 \times 10^{-10}$ ). Such a value of the $y$ parameter is close to or within the reach of the expected sensitivity of the PIXIE and the PRISM experiments. Hence if the IceCube events in the energy region of $30 \mathrm{TeV} \lesssim E \lesssim 2 \mathrm{PeV}$ are explained in the present scenario, the future experiments may have a chance to see the CMB spectral distortion.

Here, we also point out that in order to explain the IceCube events in the present scenario, $\bar{E}_{\nu}$ cannot be arbitrary large. We found that, for $\bar{E}_{\nu} \gtrsim 5 \times 10^{10} \mathrm{GeV}$, the flux of $\left.E^{2} \Phi_{\nu}(E)\right|_{E=1 \mathrm{PeV}} \sim 10^{-8} \mathrm{GeVcm}^{-2} \mathrm{~S}^{-1} \mathrm{sr}^{-1}$ cannot be obtained without conflicting the current observational constraints. Thus the mass of $X$ responsible for the IceCube events should be smaller than $\sim 10^{11} \mathrm{GeV}$.

Finally, we comment on the angular dependence of the neutrino flux. In the earlydecay scenario, the neutrino flux is isotropic, which is consistent with the IceCube result. On the contrary, it has also been discussed that the decay of dark matter may explain the 
IceCube events [5-11]. In such a scenario, the Galactic contribution dominates and large fraction of the energetic neutrinos is expected to come from the direction of the Galactic center. Denoting the angle between the Galactic center and the direction of the neutrino as $\theta$, the flux from $\theta<\pi / 2$ is roughly twice as large as that from $\theta>\pi / 2$ at the peak of the flux [4]. Future observations on the angular dependence may help to distinguish the scenarios with $\tau_{X} \ll t_{0}$ and the ones with $\tau_{X} \gg t_{0}$.

\section{Conclusions and discussion}

In this paper, we have studied the cosmological implications of high-energy neutrino injection from the decay of a massive particle $X$. When considering high-energy neutrinos in the early universe, the scattering processes with background neutrinos are important. We have numerically followed the evolution of the high-energy neutrino flux including such neutrino scattering effects, and calculate the present neutrino flux. Importantly, even only via the weak interaction, energetic neutrinos with $E \sim 10^{8}-10^{10} \mathrm{GeV}$ can effectively scatter off background neutrinos at $1+z \gtrsim 10^{5}-10^{3}$. Such scattering processes affect the shape of the cosmic-ray neutrino spectrum, as well as produce CMB distortions by the emission of photons and charged particles.

We have derived the upper bounds on the yield variable $Y_{X}$ as a function of the energy $\bar{E}_{\nu}$ of the neutrino emitted from the $X$ decay and $z_{*} \equiv z\left(\tau_{X}\right)$, using observational bounds on the high-energy cosmic-ray neutrino flux and the CMB spectral distortions. We have seen that the former gives more stringent bound for the case where the neutrino emission occurs when $1+z \lesssim 10^{4}-10^{5}$. In particular, for $1+z_{*} \lesssim 10^{4}$, the neutrino scattering processes are irrelevant, and the upper bound on $Y_{X}$ depends only on the combination of $\bar{E}_{\nu} /\left(1+z_{*}\right)$; in such a case, we found that the bounds are

- $Y_{X} \lesssim 9 \times 10^{-23}$ for $\bar{E}_{\nu} /\left(1+z_{*}\right)=10^{4} \mathrm{GeV}$,

- $Y_{X} \lesssim 2 \times 10^{-25}$ for $\bar{E}_{\nu} /\left(1+z_{*}\right)=10^{5} \mathrm{GeV}$,

- $Y_{X} \lesssim 1 \times 10^{-26}$ for $\bar{E}_{\nu} /\left(1+z_{*}\right)=10^{6} \mathrm{GeV}$,

- $Y_{X} \lesssim 8 \times 10^{-28}$ for $\bar{E}_{\nu} /\left(1+z_{*}\right)=10^{7} \mathrm{GeV}$.

On the other hand, for $1+z_{*} \gtrsim 10^{5}$, BBN gives a stronger bound. With the current accuracy, the CMB bound is less stringent than the BBN bound. However, with the sensitivity of the future experiments, PIXIE and PRISM, for example, the upper bound from CMB observation is expected to be improved by about three orders of magnitude, which will give stronger bound than the BBN.

We have also considered the possibility that the $\mathrm{PeV}$ neutrino events recently observed by IceCube originate from the decay of $X$. We have seen that the three PeV neutrino events (Bert, Ernie, and Big Bird) can be well explained within this scenario. In addition, we have seen that, when the decay of $X$ occurs at $1+z_{*} \sim 10^{4}$ and the initial energy of neutrino produced by the decay of $X$ is $\sim 10^{10} \mathrm{GeV}$, we have a possibility to realize an $E^{-2}$ power law neutrino spectrum with a cutoff at $\sim \mathrm{PeV}$, which is suggested by the IceCube 
results; for such a scenario, $z_{*}$ should be tuned with the accuracy of $O(10 \%)$. In addition, future observation of the CMB may be able to detect the distortion of the CMB spectrum caused by the decay of $X$.

We emphasize here that the mass of $X$ responsible for the $\mathrm{PeV}$ neutrino events can be as large as $O\left(10^{10} \mathrm{GeV}\right.$ ), if $1+z_{*} \sim 10^{4}-10^{5}$ (which corresponds to $\tau_{X} \sim 10^{11}-10^{9} \mathrm{sec}$ ). In other words, the IceCube experiment can probe the physics at the energy scale much higher than $\mathrm{PeV}$. One of the examples of the new physics containing the candidate of the massive particle $X$ is the model with Peccei-Quinn symmetry [48, 49] because the natural scale of the Peccei-Quinn symmetry breaking is $O\left(10^{9-10} \mathrm{GeV}\right)$. Another possibility can be a messenger sector in gauge-mediated supersymmetry breaking model [50-52]. More discussion about particle-physics models with the candidates of the massive particle $X$ is found, for example, in $[4,5,11]$. Therefore, the future IceCube experiment can shed light not only on astrophysical sources of cosmic-ray neutrinos, but also on high-energy particle-physics.

\section{Acknowledgments}

One of the authors (T.M.) is grateful to the Mainz Institute for Theoretical Physics (MITP) for its hospitality and its partial support during the completion of this work. R.J. is supported by the JSPS fellowship (No. 25-8360). T.M. is supported by the JSPS KAKENHI (No. 26400239 and No. 60322997). The work of Y.E. and R.J. is also supported by the Program for Leading Graduate Schools, MEXT, Japan.

Open Access. This article is distributed under the terms of the Creative Commons Attribution License (CC-BY 4.0), which permits any use, distribution and reproduction in any medium, provided the original author(s) and source are credited.

\section{References}

[1] A. Ibarra, D. Tran and C. Weniger, Indirect searches for decaying dark matter, Int. J. Mod. Phys. A 28 (2013) 1330040 [arXiv:1307.6434] [INSPIRE].

[2] ICECuBE collaboration, M.G. Aartsen et al., Evidence for high-energy extraterrestrial neutrinos at the IceCube detector, Science 342 (2013) 1242856 [arXiv:1311.5238] [INSPIRE].

[3] ICECuBE collaboration, M.G. Aartsen et al., Observation of high-energy astrophysical neutrinos in three years of IceCube data, Phys. Rev. Lett. 113 (2014) 101101 [arXiv: 1405.5303] [INSPIRE].

[4] Y. Ema, R. Jinno and T. Moroi, Cosmic-ray neutrinos from the decay of long-lived particle and the recent IceCube result, Phys. Lett. B 733 (2014) 120 [arXiv:1312.3501] [InSPIRE].

[5] B. Feldstein, A. Kusenko, S. Matsumoto and T.T. Yanagida, Neutrinos at IceCube from heavy decaying dark matter, Phys. Rev. D 88 (2013) 015004 [arXiv:1303.7320] [INSPIRE].

[6] A. Esmaili and P.D. Serpico, Are IceCube neutrinos unveiling PeV-scale decaying dark matter?, JCAP 11 (2013) 054 [arXiv:1308.1105] [INSPIRE].

[7] Y. Bai, R. Lu and J. Salvado, Geometric compatibility of IceCube TeV-PeV neutrino excess and its galactic dark matter origin, arXiv:1311.5864 [INSPIRE]. 
[8] A. Bhattacharya, M.H. Reno and I. Sarcevic, Reconciling neutrino flux from heavy dark matter decay and recent events at IceCube, JHEP 06 (2014) 110 [arXiv:1403.1862] [INSPIRE].

[9] J. Zavala, Galactic PeV neutrinos from dark matter annihilation, Phys. Rev. D 89 (2014) 123516 [arXiv:1404.2932] [INSPIRE].

[10] C.-H. Chen and T. Nomura, Inert dark matter in type-II seesaw, JHEP 09 (2014) 120 [arXiv: 1404.2996] [INSPIRE].

[11] T. Higaki, R. Kitano and R. Sato, Neutrinoful universe, JHEP 07 (2014) 044 [arXiv: 1405.0013] [INSPIRE].

[12] L.A. Anchordoqui et al., Cosmic neutrino Pevatrons: a brand new pathway to astronomy, astrophysics and particle physics, JHEAp 1-2 (2014) 1 [arXiv:1312.6587] [INSPIRE].

[13] D.J. Fixsen et al., Cosmic microwave background dipole spectrum measured by the COBE FIRAS, Astrophys. J. 420 (1994) 445 [INSPIRE].

[14] D.J. Fixsen et al., The Cosmic Microwave Background spectrum from the full COBE FIRAS data set, Astrophys. J. 473 (1996) 576 [astro-ph/9605054] [INSPIRE].

[15] A. Kogut et al., The Primordial Inflation Explorer (PIXIE): a nulling polarimeter for cosmic microwave background observations, JCAP 07 (2011) 025 [arXiv:1105.2044] [INSPIRE].

[16] PRISM collaboration, P. André et al., PRISM (Polarized Radiation Imaging and Spectroscopy Mission): an extended white paper, JCAP 02 (2014) 006 [arXiv:1310.1554] [INSPIRE].

[17] M.C. Gonzalez-Garcia, M. Maltoni, J. Salvado and T. Schwetz, Global fit to three neutrino mixing: critical look at present precision, JHEP 12 (2012) 123 [arXiv:1209.3023] [INSPIRE].

[18] T. Sjöstrand, S. Mrenna and P.Z. Skands, PYTHIA 6.4 physics and manual, JHEP 05 (2006) 026 [hep-ph/0603175] [INSPIRE].

[19] T. Sjöstrand, S. Mrenna and P.Z. Skands, A brief introduction to PYTHIA 8.1, Comput. Phys. Commun. 178 (2008) 852 [arXiv:0710.3820] [InSPIRE].

[20] V.B. Berestetsky, E.M. Lifshitz and L.P. Pitaevsky, Quantum electrodynamics, Oxford University Press, Oxford U.K. (1982).

[21] W. Hu and J. Silk, Thermalization constraints and spectral distortions for massive unstable relic particles, Phys. Rev. Lett. 70 (1993) 2661 [INSPIRE].

[22] X.-L. Chen and M. Kamionkowski, Particle decays during the cosmic dark ages, Phys. Rev. D 70 (2004) 043502 [astro-ph/0310473] [INSPIRE].

[23] S.R. Furlanetto, S.P. Oh and E. Pierpaoli, The effects of dark matter decay and annihilation on the high-redshift $21 \mathrm{~cm}$ background, Phys. Rev. D 74 (2006) 103502 [astro-ph/0608385] [INSPIRE].

[24] M. Valdes, A. Ferrara, M. Mapelli and E. Ripamonti, Constraining DM through $21 \mathrm{~cm}$ observations, Mon. Not. Roy. Astron. Soc. 377 (2007) 245 [astro-ph/0701301] [INSPIRE].

[25] L. Zhang, X. Chen, M. Kamionkowski, Z.-g. Si and Z. Zheng, Constraints on radiative dark-matter decay from the Cosmic Microwave Background, Phys. Rev. D 76 (2007) 061301 [arXiv: 0704.2444] [INSPIRE].

[26] J. Chluba and R.A. Sunyaev, The evolution of CMB spectral distortions in the early universe, Mon. Not. Roy. Astron. Soc. 419 (2012) 1294 [arXiv:1109.6552] [InSPIRE]. 
[27] T.R. Slatyer, Energy injection and absorption in the cosmic dark ages, Phys. Rev. D 87 (2013) 123513 [arXiv:1211.0283] [INSPIRE].

[28] R. Khatri and R.A. Sunyaev, Beyond $y$ and $\mu$ : the shape of the CMB spectral distortions in the intermediate epoch, $1.5 \times 10^{4}<z<2 \times 10^{5}$, JCAP 09 (2012) 016 [arXiv:1207.6654] [INSPIRE].

[29] J. Chluba, Green's function of the cosmological thermalization problem, Mon. Not. Roy. Astron. Soc. 434 (2013) 352 [arXiv:1304.6120] [InSPIRE].

[30] J. Chluba, Distinguishing different scenarios of early energy release with spectral distortions of the cosmic microwave background, Mon. Not. Roy. Astron. Soc. 436 (2013) 2232 [arXiv: 1304.6121] [INSPIRE].

[31] J. Chluba and D. Jeong, Teasing bits of information out of the CMB energy spectrum, arXiv:1306.5751 [INSPIRE].

[32] Y.B. Zeldovich and R.A. Sunyaev, The interaction of matter and radiation in a hot-model universe, Astrophys. Space Sci. 4 (1969) 301 [INSPIRE].

[33] R.A. Sunyaev and Y. Zeldovich, The interaction of matter and radiation in the hot model of the universe, Astrophys. Space Sci. 7 (1970) 20 [INSPIRE].

[34] L. Danese and G. de Zotti, Double Compton process and the spectrum of the microwave background, Astron. Astrophys. 107 (1982) 39.

[35] C. Burigana, G. de Zotti and L. Danese, Constraints on the thermal history of the universe from the cosmic microwave background spectrum, Astrophys. J. 379 (1991) 1.

[36] W. Hu and J. Silk, Thermalization and spectral distortions of the cosmic background radiation, Phys. Rev. D 48 (1993) 485 [INSPIRE].

[37] C. Burigana, G. de Zotti and L. Danese, Analytical description of spectral distortions of the cosmic microwave background, Astron. Astrophys. 303 (1995) 323.

[38] J. Chluba, R. Khatri and R.A. Sunyaev, CMB at $2 \times 2$ order: the dissipation of primordial acoustic waves and the observable part of the associated energy release, Mon. Not. Roy. Astron. Soc. 425 (2012) 1129 [arXiv:1202.0057] [inSPIRE].

[39] T.R. Slatyer, N. Padmanabhan and D.P. Finkbeiner, CMB constraints on WIMP annihilation: energy absorption during the recombination epoch, Phys. Rev. D 80 (2009) 043526 [arXiv:0906.1197] [InSPIRE].

[40] M. Kawasaki, K. Kohri and T. Moroi, Hadronic decay of late-decaying particles and Big-Bang nucleosynthesis, Phys. Lett. B 625 (2005) 7 [astro-ph/0402490] [InSPIRE].

[41] M. Kawasaki, K. Kohri and T. Moroi, Big-Bang nucleosynthesis and hadronic decay of long-lived massive particles, Phys. Rev. D 71 (2005) 083502 [astro-ph/0408426] [INSPIRE].

[42] M. Kawasaki and T. Moroi, Gravitino decay into a neutrino and a sneutrino in the inflationary universe, Phys. Lett. B 346 (1995) 27 [hep-ph/9408321] [INSPIRE].

[43] T. Kanzaki, M. Kawasaki, K. Kohri and T. Moroi, Cosmological constraints on neutrino injection, Phys. Rev. D 76 (2007) 105017 [arXiv: 0705.1200] [INSPIRE].

[44] M. Honda, T. Kajita, K. Kasahara, S. Midorikawa and T. Sanuki, Calculation of atmospheric neutrino flux using the interaction model calibrated with atmospheric muon data, Phys. Rev. D 75 (2007) 043006 [astro-ph/0611418] [INSPIRE]. 
[45] ICECuBE collaboration, R. Abbasi et al., A search for a diffuse flux of astrophysical muon neutrinos with the IceCube 40-string detector, Phys. Rev. D 84 (2011) 082001 [arXiv:1104.5187] [INSPIRE].

[46] ICECube collaboration, M.G. Aartsen et al., Probing the origin of cosmic-rays with extremely high energy neutrinos using the IceCube observatory, Phys. Rev. D 88 (2013) 112008 [arXiv: 1310.5477] [INSPIRE].

[47] C.-Y. Chen, P.S. Bhupal Dev and A. Soni, Standard model explanation of the ultrahigh energy neutrino events at IceCube, Phys. Rev. D 89 (2014) 033012 [arXiv:1309.1764] [InSPIRE].

[48] R.D. Peccei and H.R. Quinn, CP conservation in the presence of instantons, Phys. Rev. Lett. 38 (1977) 1440 [INSPIRE].

[49] R.D. Peccei and H.R. Quinn, Constraints imposed by CP conservation in the presence of instantons, Phys. Rev. D 16 (1977) 1791 [INSPIRE].

[50] M. Dine and A.E. Nelson, Dynamical supersymmetry breaking at low-energies, Phys. Rev. D 48 (1993) 1277 [hep-ph/9303230] [InSPIRE].

[51] M. Dine, A.E. Nelson and Y. Shirman, Low-energy dynamical supersymmetry breaking simplified, Phys. Rev. D 51 (1995) 1362 [hep-ph/9408384] [INSPIRE].

[52] M. Dine, A.E. Nelson, Y. Nir and Y. Shirman, New tools for low-energy dynamical supersymmetry breaking, Phys. Rev. D 53 (1996) 2658 [hep-ph/9507378] [INSPIRE]. 